\title{
Mitigation of Signal Distortions Using Reference Signal Distribution With Colorless Remote Antenna Units for Radio-Over-Fiber Applications
}

\author{
C. W. Chow, Member, IEEE, L. Xu, Student Member, IEEE, C. H. Yeh, C. H. Wang, Student Member, IEEE,
} F. Y. Shih, Student Member, IEEE, H. K. Tsang, Senior Member, IEEE, C. L. Pan, Senior Member, IEEE, Fellow, OSA, and S. Chi, Fellow, OSA

\begin{abstract}
In this paper, we propose and demonstrate a bidirectional radio-over-fiber $(\mathrm{ROF})$ system using a reference signal distribution. In order to minimize the radio-frequency (RF) signal attenuation as well as limit the transmit power to moderate levels, small cell size, or picocell is used in the ROF system. This will result in the need of many base stations (BSs) to provide sufficient network coverage. Because of this, colorless remote antenna unit (RAU) with optical carrier generated and distributed from the head end (HE) is employed. Experimental results show that error-free signal transmission and remodulation using downstream differential phase shift keying (DPSK) and upstream ON-OFF keying (OOK) is achieved in a $20-\mathrm{km}$ reach ROF system. Experimental characterization of the reflective semiconductor optical amplifier (RSOA)-based remodulation unit is also performed. Numerical simulation is performed to compare the proposed scheme with several conventional optical millimeter-wave (mm-wave) schemes, including double sideband (DSB), optical carrier suppression (OCS), and single sideband (SSB). Results show that the proposed scheme could be a potential candidate to mitigate signal distortions due to signal fading and code time shifting.
\end{abstract}

Index Terms-Hybrid wired/wireless network, signal remodulation, radio-over-fiber (ROF), optical millimeter-wave (mm-wave).

\section{INTRODUCTION}

$\mathbf{T}$ HE convergence of wireless and optical fiber systems in an integrated platform has become a promising technique in future Internet for providing broadband wired and wireless access services with increased mobility and reduced cost

Manuscript received November 30, 2008; revised May 26, 2009 and June 21, 2009. First published July 07, 2009; current version published September 10, 2009. This work was supported by the National Science Council, Taiwan, under Contracts NSC 96-2218-E-009-025-MY2 and 97-2221-E-009-038-MY3.

C. W. Chow, C. H. Wang, F. Y. Shih, and S. Chi are with the Department of Photonics, National Chiao Tung University, Hsinchu 30010, Taiwan (e-mail: cwchow@faculty.nctu.edu.tw; shoutclose@gmail.com; yuan0253@ hotmail. com; schi@mail.nctu.edu.tw).

L. Xu and H. K. Tsang are with the Department of Electronic Engineering, The Chinese University of Hong Kong, Shatin, Hong Kong (e-mail: 1xu@ee. cuhk.edu.hk; hktsang@ee.cuhk.edu.hk).

C. H. Yeh is with the Information and Communications Research Laboratories, Industrial Technology Research Institute, Hsinchu 31040, Taiwan (e-mail: depew@itri.org.tw).

C. L. Pan was with the Department of Photonics, National Chiao-Tung University, Hsinchu, Taiwan. He is now with the Department of Physics and Institute of Photonics Technologies, National Tsing-Hua University, Hsinchu 30013, Taiwan (e-mail: clpan@phys.nthu.edu.tw).

Color versions of one or more of the figures in this paper are available online at http://ieeexplore.ieee.org.

Digital Object Identifier 10.1109/JLT.2009.2026654
[1]-[6]. For the broadband wired system, passive optical network $(\mathrm{PON})$ is considered as a promising candidate to replace the copper-based approaches due to its high data throughput and cost effectiveness [7]-[13]. The first generations of gigabit PONs (GPON) have now been standardized and typically offer $1-2.5 \mathrm{~Gb} / \mathrm{s}$ downstream and $\sim 1 \mathrm{~Gb} / \mathrm{s}$ upstream. For the wireless communication, it is now entering a new phase where the focus is moving from voice to high bandwidth (BW) multimedia services. In order to support high data rate wireless communication in the future, wireless system would utilize millimeter-wave (mm-wave) as the signal carrier. We can see that many research efforts have been made at the $60-\mathrm{GHz}$ band [14]-[19] or above [20]. However, due to the relatively high atmospheric attenuation in the mm-wave frequency band, and maintaining the high signal-to-noise ratio (SNR) at the receiver (Rx) while limiting the transmit power to moderate levels, each mm-wave base station (BS) has to reduce the cell size (picocell) [21]. The use of picocells in the wireless communication system will result in the need of many BSs to provide sufficient network coverage, and thus greatly increase the cost of the overall system. In addition, the small cell size will increase the network burden during mobile state (MS) hand-off, since the users could travel through several picocells in a short period of time.

Because of this, radio-over-fiber (ROF) system [22]-[27] using BS to extend radio-frequency (RF) signal connection and distribution has been proposed to simplify the network architecture and reduce the cost. Optical fiber is an excellent medium for RF signal transmission due to the very high BW, low loss, light weight, small cross section, and low cost. In this ROF system, a head-end (HE), which consists of an optical-to-electrical $(\mathrm{O} / \mathrm{E})$ and an electrical-to-optical (E/O) modules, is connected to the BS. A remote antenna unit (RAU) will be used in each picocell. Many picocells can be connected to the HE by optical fibers. In order to improve the utilization of the fiber BW and increase the network coverage, wavelength division multiplexing (WDM) can be used.

The generation and transmission of optical mm-wave are crucial in ROF systems. When the signals that are carried by the optical $\mathrm{mm}$-wave are propagating in optical fiber, chromatic dispersion causes signal distortions. The double-sideband (DSB) optical mm-wave signal suffers both the fading effect (fading leads to cosine-like signal power fluctuation along the fiber) and the time-shifting of data codes [28], [29]. Optical carrier suppression (OCS) optical mm-wave signals have been 


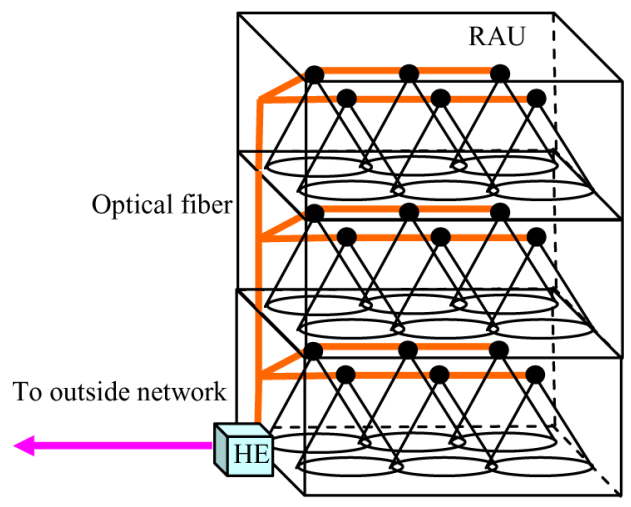

Fig. 1. Schematic ROF architecture inside a building.

proposed [28], [29] to mitigate the fading effect. However, code time shifting is severe, and distorts the signal leading to eye closure after transmission. Single-sideband (SSB) optical mm-wave may reduce the code time shifting, however the immunity depends on the generation methods [29], [30].

In this work, we propose and demonstrate a $80-\mathrm{GHz}$ bidirectional ROF link with reference signal distribution. As mentioned before, due to a large number of RAUs required for the mm-wave ROF system, colorless RAU with optical carrier generated and distributed from the HE is employed. This can reduce the inventory cost, since wavelength specific or wavelength tunable light source is not required at the RAU. Centralized wavelength control is used at the HE. In the experience, reflective semiconductor optical amplifier (RSOA) is used as the colorless modulator inside the RAU. RSOA is generally considered as a potential low-cost and relatively high data rate device. It has low polarization-dependent gain. Recently, 10-Gb/s RSOA has been implemented [31]. In the proposed scheme, the upstream data is modulated onto the downstream data by wavelength reuse [32]-[34]. Transmission comparison with the DSB, OCS, and SSB optical mm-wave signals is performed by means of numerical simulations, showing the proposed system with reference signal distribution may mitigate signal fading and code time shifting.

This paper is organized as follows. In Section II, we will describe the architecture for the proposed ROF system. Section III reports the experimental results. In Section IV, we will numerically study the performance of the baseband signal and the RF down-converted signal at different atmospheric attenuations. In Section V, we will compare the proposed scheme with the DSB, OCS, and SSB optical mm-wave signals. Finally, conclusions are given in Section VI.

\section{ARCHITECTURE}

Fig. 1 shows the schematic of a typical picocellular system inside a building. The fiber infrastructure connects many RAUs to the HE. The RAU merely consists of a simple electronic control circuit and a pair of $\mathrm{O} / \mathrm{E}$ and $\mathrm{E} / \mathrm{O}$ converter. For the downstream communication, the electrical data will be first converted to optical signal by the E/O converter at the HE. The optical data will then be transmitted to the RAU, where the O/E module will convert the optical data to electrical data, which will then be emitted by the antenna to the MS. For the upstream traffic, the $\mathrm{E} / \mathrm{O}$ converter at the RAU will convert the received RF signal

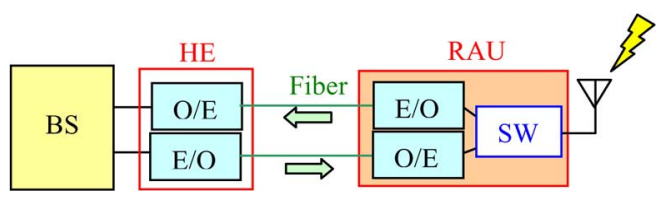

Fig. 2. Schematic of the ROF system.

from the antenna to optical signal. The signal will detected by the O/E converter at the HE. In order to further reduce the cost of the RAU, a single antenna with a switch (SW) can be used for both upstream and downstream communications by using the time-division duplex (TDD) mode (Fig. 2).

Fig. 3 shows the proposed architecture of the ROF system using reference signal distribution. For the downstream link, the optical reference signal is generated by modulating the continuous wave (CW) via a Mach-Zehnder modulator (MZM) at the HE. In order to produce the optical two tone for optical $\mathrm{mm}$-wave generation, the direct current (dc) bias of the MZM is set to a minimum transmission and driven by a $20-\mathrm{GHz}$ sinusoidal signal. Hence, DSB with suppressed carrier optical modulation is achieved, and the first-order sidebands with frequency separation equal to twice the modulating signal frequency is observed at the output of the MZM. The downstream data is encoded using differential phase-shift keying (DPSK) modulation via a phase modulator (PM). The downstream data can be used for both wireless and wired optical communication. It is transmitted at another wavelength, which is $100 \mathrm{GHz}$ away from the wavelength of the reference signal. To improve the coherence of the laser courses, dual-wavelength laser [35] or phase-locked laser (PLL) with mode spacing of $100 \mathrm{GHz}$ can be used. Both the optical reference signal and the downstream signal are wavelength multiplexed by a standard arrayed waveguide grating (AWG) [Gaussian-shaped, 100-GHz channel spacing with $3-\mathrm{dB}$ width of $50 \mathrm{GHz}]$. In this scheme, multiple ROF channels can by supported by using alternative reference and DPSK data channels aligning with the AWG passband as shown in the inset of Fig. 3.

The downstream data signal and the optical reference signal are then transmitted through a standard single-mode fiber (SMF) to the remote node (RN), which then distribute the data to different RAUs. The optical reference and the downstream signals are wavelength demultiplexed at the RN. The optical reference signal can be power divided via a passive fiber splitter to an optical $\mathrm{Rx}_{1}$ with $\mathrm{BW}$ of $40 \mathrm{GHz}$ at the $\mathrm{RAU}$ to retrieve the $40-\mathrm{GHz}$ electrical clock source, which could be used for the upstream data down-conversion from RF to baseband.

At the RAU, a high BW ( $>80 \mathrm{GHz}$ ) optical $\mathrm{Rx}_{2}$ is used to obtain the electrical mm-wave signal for the antenna. The electrical mm-wave is produced by beating the optical reference signal and the downstream demodulated DPSK signal inside the optical $\mathrm{Rx}_{2}$. For the wireless communication, a bandpass filter (BPF) with center frequency at $80 \mathrm{GHz}(\mathrm{BW}$ of $20 \mathrm{GHz}$ ) is used after the $\mathrm{Rx}_{2}$ to retrieve the mm-wave signal. A low-pass filter (LPF) with 3-dB bandwidth of $10 \mathrm{GHz}$ can be used to retrieve the $10-\mathrm{Gb} / \mathrm{s}$ optical data for wired communication. As shown in Fig. 3, the downstream DPSK signal is power divided into two equal parts. One part is launched into a delayed interferometer (DI) with 100-ps delay for demodulating the $10-\mathrm{Gb} / \mathrm{s}$ 


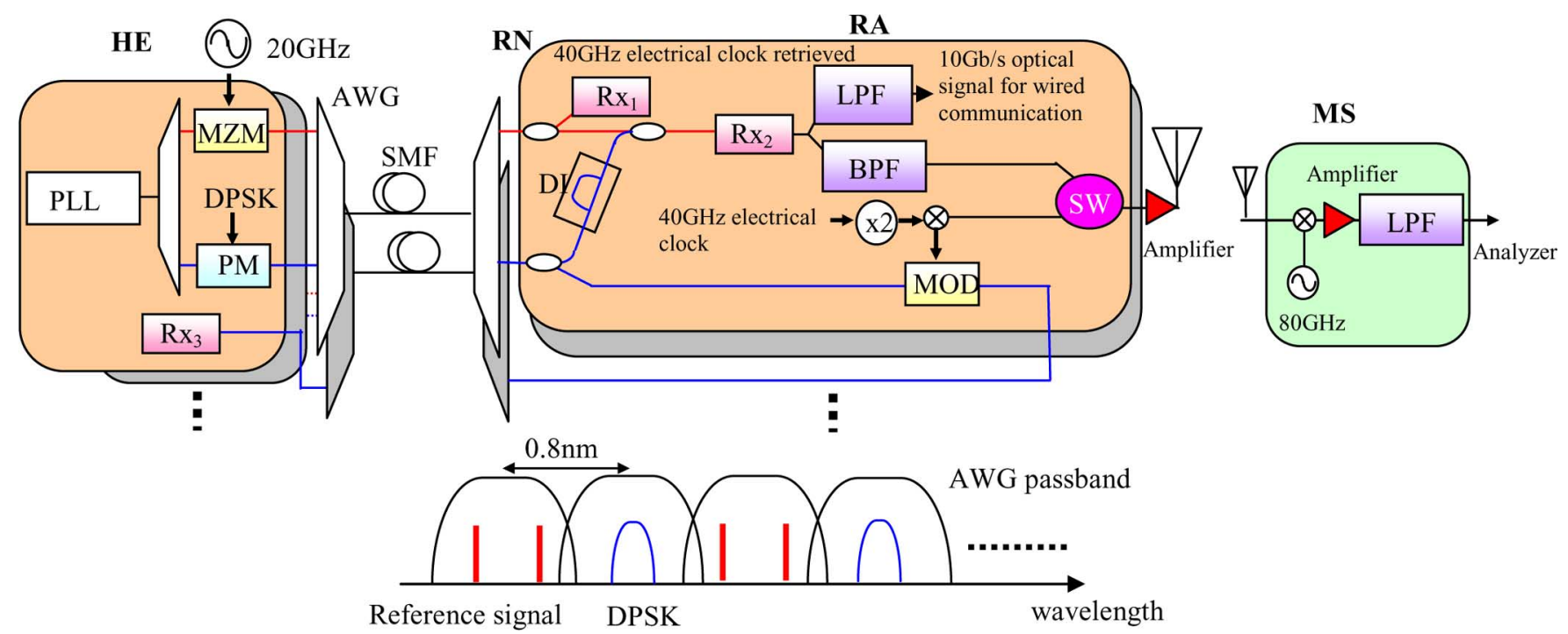

Fig. 3. Proposed ROF system using reference signal distribution. Inset: schematic of optical spectra of optical clock and DPSK signals.

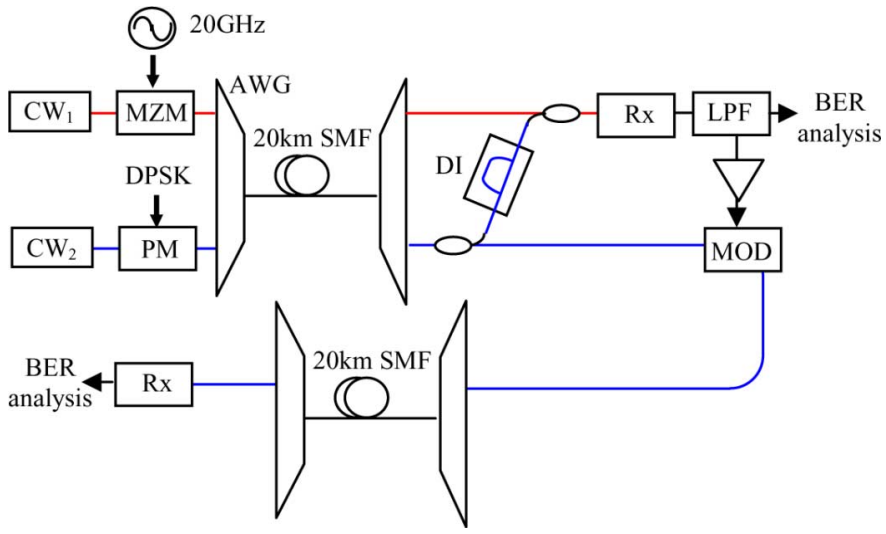

Fig. 4. Experimental setup of ROF system using a separate optical clock distribution.

DPSK signal into intensity modulated signal. The other half of the downstream signal is then launched into an intensity modulator (MOD), which is driven by a baseband nonreturn-to-zero (NRZ) upstream data for signal remodulation. The upstream NRZ ON-OFF keying (OOK) signal will then be transmitted back to the $\mathrm{Rx}_{3}$ at the HE. The baseband NRZ data can be obtained by mixing the clock signal (retrieved from the optical reference signal as described before) and the received upstream signal from the antenna. A single antenna with an electrical SW is used for both upstream and downstream communications in TDD mode.

\section{EXPERIMENT}

Fig. 4 shows the experimental setup of the ROF system. The downstream DPSK signal was modulated at $10 \mathrm{~Gb} / \mathrm{s}$, pseudorandom binary sequence (PRBS), $2^{23}-1$ at one wavelength which is $100 \mathrm{GHz}$ away from the wavelength of the optical reference signal). Both the optical reference and the DPSK signals were then transmitted through 20-km SMF without dispersion compensation. At the RAU, a $100-\mathrm{GHz}$ channel spacing, Gaussian-shaped AWG was used to wavelength demultiplexed the optical reference and the DPSK signals. The
DPSK signal was power divided by a 3-dB fiber splitter. One part was demodulated by the DI of 100 ps. The demodulated DPSK signal was then combined with the optical reference signal and launched into the optical Rx. An optical Rx with LPF bandwidth of $10 \mathrm{GHz}$ was used to obtain the $10-\mathrm{Gb} / \mathrm{s}$ demodulated DPSK data for wired communication. Due to the unavailability of $>80-\mathrm{GHz}$ optical $\mathrm{Rx}$ and the $80-\mathrm{GHz}$ electrical BPF, numerical simulation was used for the characterization; and this will be reported in Sections IV and V.

The other part of the undemodulated DPSK signal was launched into the MOD, which was an MZM used to produce the $10-\mathrm{Gb} / \mathrm{s}$ upstream optical OOK data. We also considered the case of asymmetric downstream and upstream transmissions. In this case, the downstream optical signal was then launched into a reflective semiconductor optical amplifier (RSOA), which was driven by a baseband $2.5-\mathrm{Gb} / \mathrm{s}$ NRZ upstream data for signal remodulation. The upstream OOK signal was then transmitted back to the Rx at HE.

Fig. 5 shows the bit error rate (BER) measurements of the downstream and upstream signals at back-to-back and after 20-km transmission without dispersion compensation. Fig. 5(a)-(c) shows the experimental eye diagrams of $10-\mathrm{Gb} / \mathrm{s}$ downstream demodulated DPSK signal (using the $10-\mathrm{GHz}$ electrical BPF to remove the high-frequency components detected by the Rx at RAU), upstream remodulated $10-\mathrm{Gb} / \mathrm{s}$ OOK signal, and upstream remodulated $2.5-\mathrm{Gb} / \mathrm{s}$ OOK signal using RSOA. We observed about 1.7-dB power penalty in the downstream demodulated DPSK signal. As described before, half of the downstream signal was launched into the RSOA for signal remodulation to produce the upstream signal. Due to the constant intensity of the downstream DPSK signal, only a small power penalty of $0.5 \mathrm{~dB}$ was observed in the upstream OOK signal after the $20-\mathrm{km}$ transmission. The target $10-\mathrm{Gb} / \mathrm{s}$ signal carried by mm-wave at $80-\mathrm{GHz}$ frequency band could be obtained by using an electrical BPF with center frequency of $80 \mathrm{GHz}$ and $\mathrm{BW}$ of $20 \mathrm{GHz}$ connected to a high-speed optical Rx. Numerical simulation will be described in next section.

We also studied the different operation conditions of the RSOA used at the RAU. Fig. 6(a) shows the Rx sensitivities 


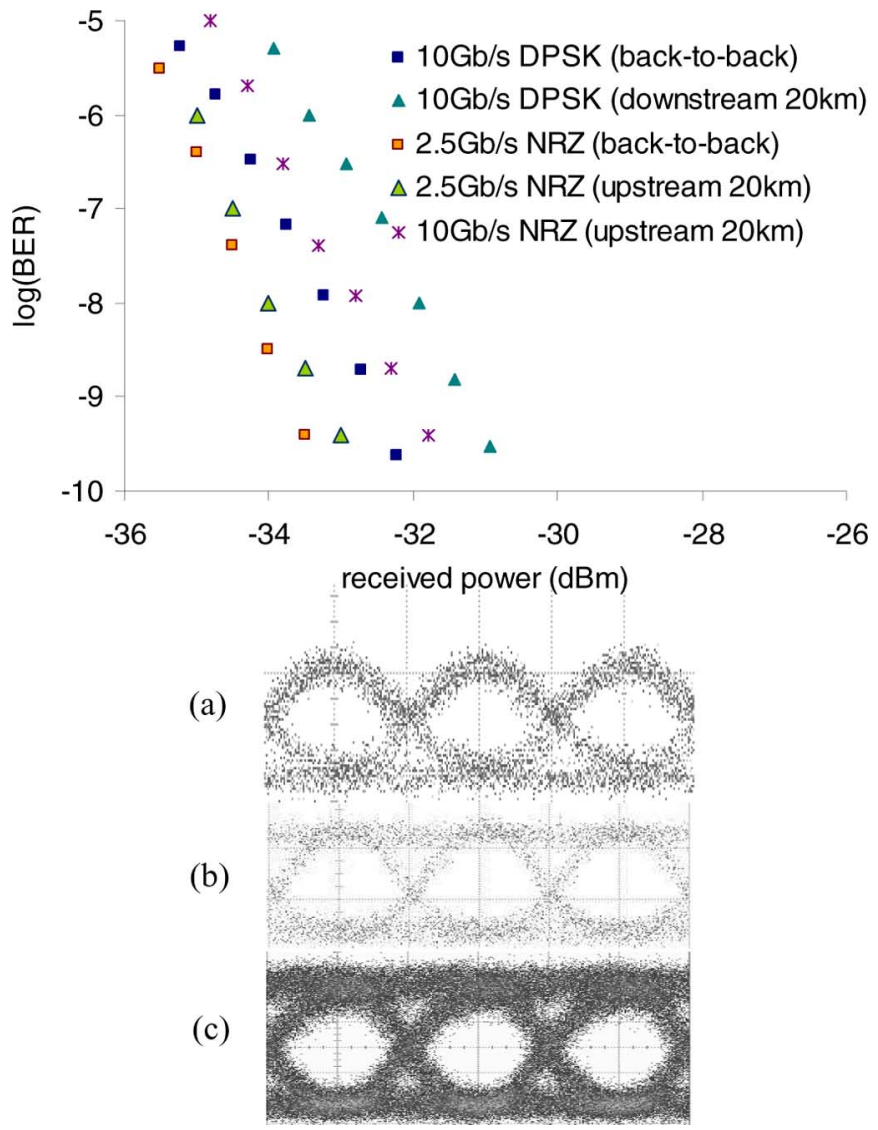

Fig. 5. BER of the 10-Gb/s downlink DPSK, 10- and 2.5-Gb/s NRZ remodulated signals. Experimental eye diagrams: (a) demodulated DPSK signal received at RAU, (b) remodulated 10-Gb/s uplink NRZ signal received at HE, and (c) remodulated $2.5 \mathrm{~Gb} / \mathrm{s}$ uplink NRZ signal received at $\mathrm{HE}$.

of the upstream OOK signal under different launched powers when the RSOA was dc biased at $95 \mathrm{~mA}$. We can observe that the $\mathrm{Rx}$ sensitivity is minimum, and starts to saturate at launched power of $-2 \mathrm{dBm}$. Rx sensitivity of $-23 \mathrm{dBm}$ was measured when the input power to the RSOA is about $-10 \mathrm{dBm}$. Fig. $6(\mathrm{~b})$ shows the Rx sensitivities of the upstream OOK signal under different dc biases, when the input downstream signal to the RSOA is at $\sim 0 \mathrm{dBm}$. We can also see that the optimum dc driving current to the RSOA is $\geq 85 \mathrm{~mA}$. It is also worth mentioning that synchronization between the downstream and remodulation upstream signals is not required. Fig. 6(c) shows the measured Rx sensitivities of the upstream OOK signal under different delays of the downstream DPSK signal when the RSOA was dc based at $95 \mathrm{~mA}$ and the input optical power was about $0 \mathrm{dBm}$.

We also experimentally characterized our 40-GHz optical reference signal (clock signal) by studying the SSB noise spectrum (Fig. 7). In this measure, the optical Rx was a $40-\mathrm{GHz}$ PIN photodiode connected to an RF spectrum analyzer (HP8564E). The SSB noise spectrum of an electrical $40-\mathrm{GHz}$ signal generated by an RF frequency synthesizer (Agilent E8244A) was also given for comparison. We observed that the SSB noise of the 40-GHz signal generated by the optical means shows similar performance when compared with the frequency synthesizer. It is about $-95 \mathrm{dBc} / \mathrm{Hz}$ at the spot frequency of $10-\mathrm{kHz}$ offset.
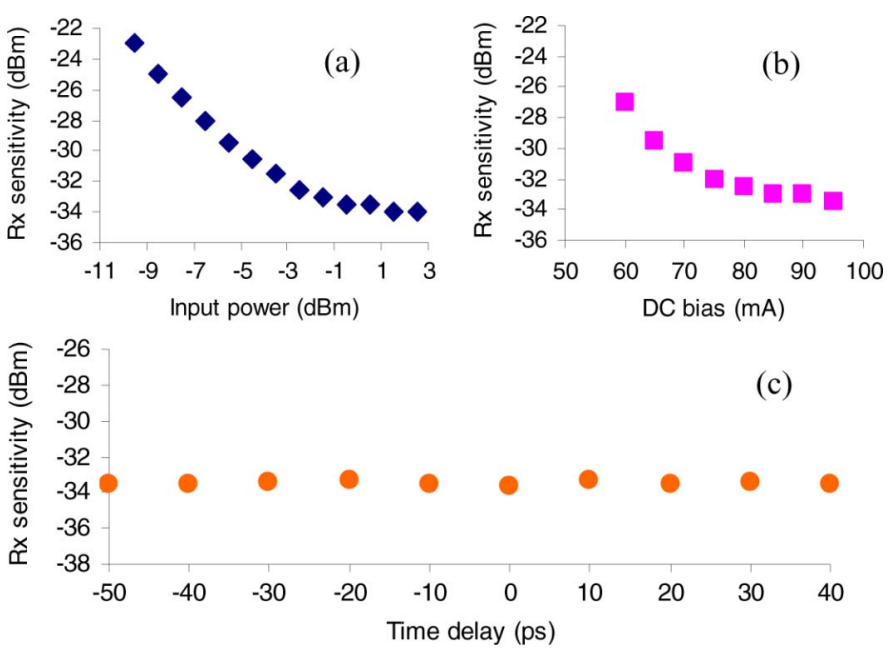

Fig. 6. Rx sensitivities of the upstream remodulated OOK signal (a) under different injected powers when the RSOA was dc biased at $95 \mathrm{~mA}$, (b) under different dc biases, when the input downstream signal to the RSOA is at $\sim 0 \mathrm{dBm}$, and (c) under different delays of the downstream DPSK signals.

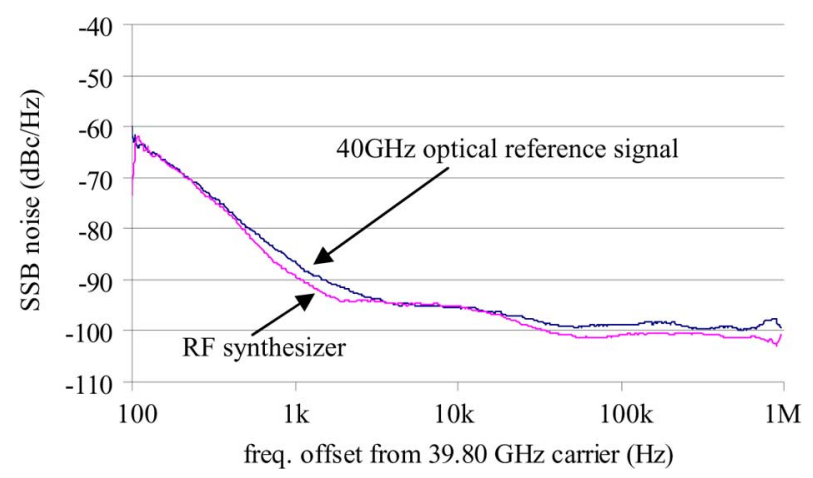

Fig. 7. SSB noise spectrum of the RF frequency synthesizer measured at $40-\mathrm{GHz}$ tone and the $40-\mathrm{GHz}$ optical reference signal.

\section{NUMERICAL ANALYSIS}

Numerical simulations were performed using VPI Transmission Maker V7.5. For our proposed scheme, we used a dualwavelength PLL with frequency separation of $100 \mathrm{GHz}$. The laser linewidth is $10 \mathrm{MHz}$. DPSK signal of $10 \mathrm{~Gb} / \mathrm{s}$ was encoded at one wavelength. On the other wavelength, the optical reference signal was generated by biasing an MZM at transmission minimum and driven by a $20-\mathrm{GHz}$ sinusoidal signal. Hence, DSB with suppressed carrier optical modulation was achieved. Both signals are wavelength multiplexed by an AWG (Gaussian-shaped, 100-GHz channel spacing with 3-dB width of $50 \mathrm{GHz}$ ). They were then transmitted through $20 \mathrm{~km} \mathrm{SMF}$ (dispersion parameter $=17 \mathrm{ps} / \mathrm{nm} / \mathrm{km}$ ). They were wavelength demultiplexed by another AWG. Half power of the DPSK signal was demodulated by the DI of 100 ps. The demodulated DPSK signal was then combined using fiber coupler with the optical reference signal, and launched into the optical Rx.

Fig. 8(a) shows the simulated optical spectra of the data and reference signals and Fig. 8(b) shows the simulated RF spectra of different electrical components detected at the downstream Rx inside the RAU. In Fig. 8(b), we can observe the baseband $10-\mathrm{Gb} / \mathrm{s}$ data, a $40-\mathrm{GHz}$ tone (generated by the reference signal 


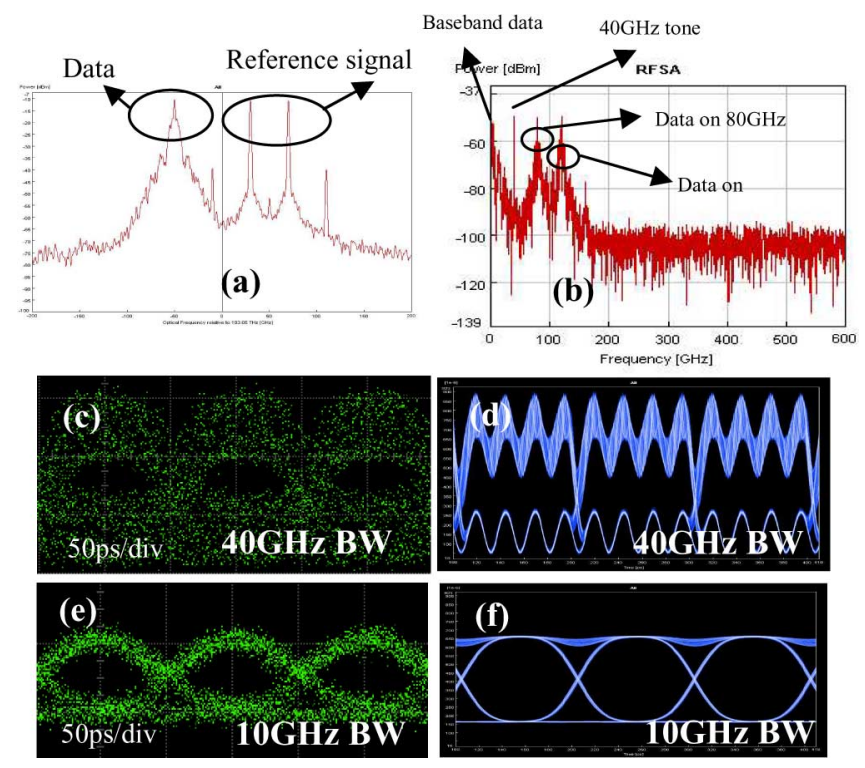

Fig. 8. (a) Simulated optical spectra of the DPSK and reference signals. (b) Simulated RF spectra of different electrical components detected at the downstream Rx inside the RAU. Eye diagrams: (c) experimental and (d) simulated downlink signal measured by Rx with a 40-GHz LPF; (e) experimental and (f) simulated downlink signal measured by Rx with 10-GHz LPF.

itself), a $10-\mathrm{Gb} / \mathrm{s}$ data on $80-\mathrm{GHz}$ carrier (generated by the coherent beating between the DPSK and the lower sideband of the reference signal), a 10-Gb/s data on $120-\mathrm{GHz}$ carrier (generated by the coherent beating between the DPSK and the upper sideband of the reference signal). We used a 40-GHz bandwidth optical $\mathrm{Rx}$ in the experiment to confirm the photomixing processing. Fig. 8(c) and (d) shows the experimental and simulated eye diagrams of the optical mm-wave signal detected at the RAU (Fig. 3) by using Rx with an LPF bandwidth of $40 \mathrm{GHz}$. We observed a good match in eye-shape between them. Since the BER tester and the RF synthesizer were not synchronized, we cannot observe the sinusoidal signal at the top and bottom of the experimental eye in Fig. 8(c). Then, by inserting an electrical LPF with BW of $10 \mathrm{GHz}$ after the optical Rx, the baseband $10-\mathrm{Gb} / \mathrm{s}$ intensity modulated signal can be clearly observed by removing the high-frequency components. Fig. 8(e) and (f) shows the experimental and simulated eye diagrams of the baseband $10-\mathrm{Gb} / \mathrm{s}$ signal, respectively.

Then, simulations were performed for the RF signal $(10-\mathrm{Gb} / \mathrm{s}$ data on $80-\mathrm{GHz}$ carrier) analysis. Two different optical launched powers to the $\mathrm{Rx}_{2}$ inside the RAU in Fig. $3(-31$ and $-28 \mathrm{dBm}$ ) were studied. Launched power of $-31 \mathrm{dBm}$ was chosen due to the error-free demodulated DPSK condition as shown in Fig. 5. RF amplifiers (20-dB gain, noise $3 \mathrm{~dB}$ ) were used in the RAU and the MS, respectively. Fig. 9 shows the simulated Q (decibels) values of the down-converted OOK signal at the MS under different atmospheric attenuations between the RAU and the MS, showing the atmospheric attenuation can up to $55 \mathrm{~dB}$ for the optical input power of $-31 \mathrm{dBm}$ to the RAU, in order to achieve error-free down-converted signal at the MS. Other signal fading effects between the RAU and MS were not included in the simulation since this is out of the scope of this paper.

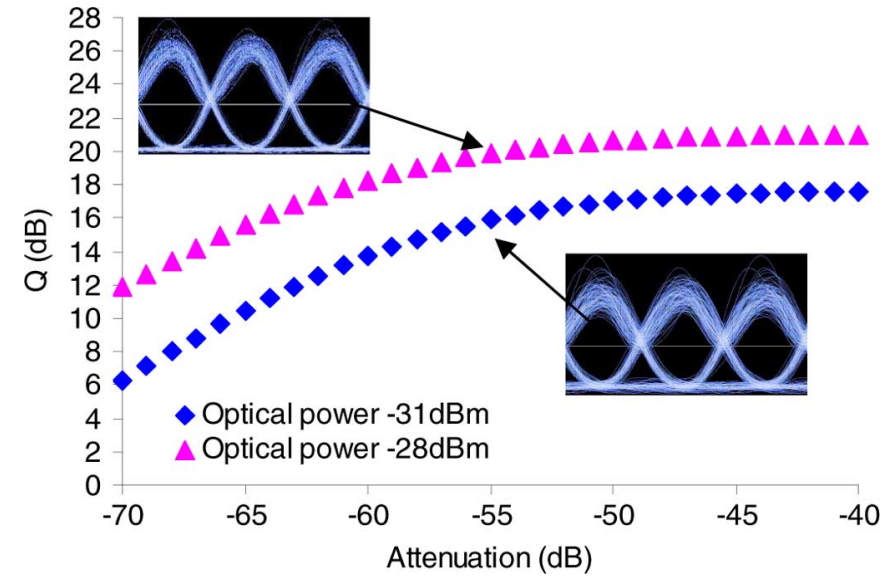

Fig. 9. Simulated Q (decibels) of the down-converted BPSK signal at the MS under different attenuations between the RAU and the MS. Insets: simulated eyes of demodulated OOK at MS when attenuation was $-55 \mathrm{~dB}$.

\section{COMPARISON WITH DSB, OCS, AND SSB SCHEMES}

In this section, we compared the proposed scheme with the DSB, OCS, and SSB optical mm-wave schemes by using VPI Transmission Maker V7.5. To simplify the comparison, $10-\mathrm{Gb} / \mathrm{s}$ NRZ baseband data modulated on a $80-\mathrm{GHz}$ carrier was used in all cases. The simulation parameters of the proposed scheme were described in Section IV. Since the performances of the optical mm-wave signals depend on the optical generation methods, we arbitrary divided the generation methods into two categories. Fig. 10 shows the first category of optical $\mathrm{mm}$-wave generation using DSB, SSB, and OCS as described in [26], with the corresponding simulated output optical spectra (resolution $0.01 \mathrm{~nm}$ ). Baseband data signal was generated by the first MZM driven by a $10-\mathrm{Gb} / \mathrm{s}$ electrical NRZ signal. For the DSB modulation scheme, the second MZM was biased at $0.5 \mathrm{~V}_{\pi}$, and the frequency of the driven RF signal was $80 \mathrm{GHz}$. A dual-arm MZM was used to achieve the SSB modulation, as shown in Fig. 9(b). The two electrical RF signals to drive the dual-arm MZM had a $\pi / 2$ phase shift, and was dc biased at $0.5 \mathrm{~V}_{\pi}$. When the phase difference of the two electrical RF signals to drive the dual-arm MZM was $\pi$, and the dc bias was at $\mathrm{V}_{\pi}$, OCS modulation is achieved, as shown in Fig. 9(c). All the signals were detected by using optical Rx with Bessel third-order BPF at center frequency of $80 \mathrm{GHz}$ and $\mathrm{BW}$ of $20 \mathrm{GHz}$.

As shown in Fig. 10(a), for the DSB optical mm-wave, the electrical current in the Rx is generated by three lightwaves at $\omega_{c}-\omega_{m}, \omega_{c}, \omega_{c}+\omega_{m}$, where the $\omega_{c}$ and $\omega_{m}$ are the frequencies of the optical carrier and the modulation, respectively. Hence, it suffers from both fading and the code time-shifting [Fig. 11(a)]. In this category of generation methods, we can observe from Fig. 10 that the optical spectra of the SSB and OCS are similar. There are two dominant tones in each signal $\left(\omega_{c}-\omega_{m}\right.$ and $\omega_{c}$, in the SSB modulation; and $\omega_{c}-\omega_{m} / 2$ and $\omega_{c}+\omega_{m} / 2$ in the OCS modulation). The transmission distance of the SSB and OCS are limited due to the code time shifting by the two dominant tones, as shown in Fig. 11(b) and (c). For the proposed approach as shown in Fig. 11(d), we can observe that the envelope of the signal can maintain the shape. 
(a)

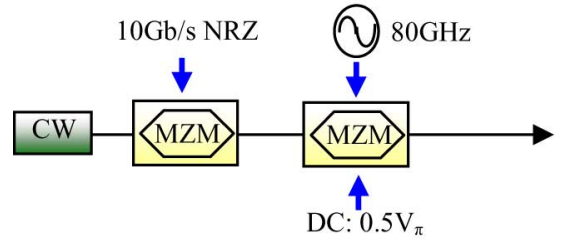

(b)

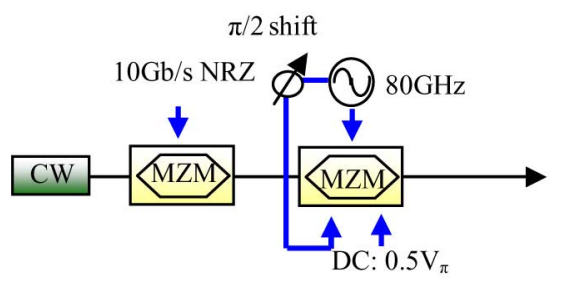

(c)

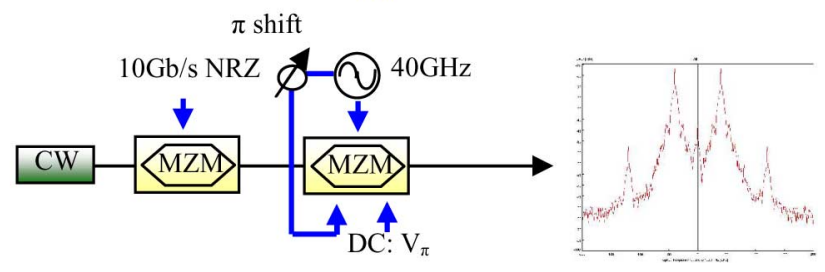

Fig. 10. Up-conversions of the DSB, SSB, and OCS [26]. (a) DSB. (b) SSB. (c) OCS.

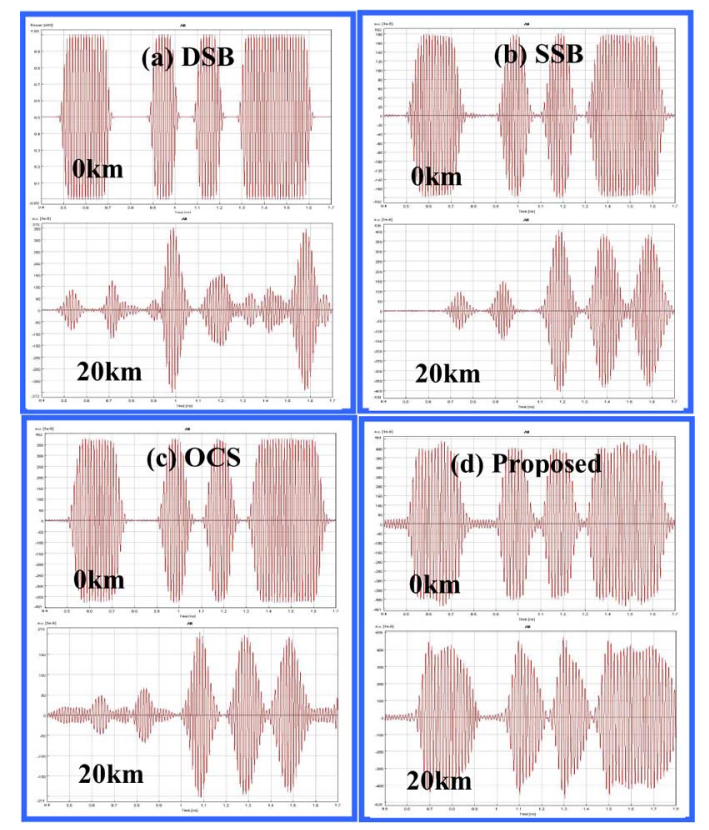

Fig. 11. Simulated time traces (first category) of $10-\mathrm{Gb} / \mathrm{s} \mathrm{NRZ} \mathrm{mm-wave} \mathrm{signal}$ modulated by $80-\mathrm{GHz}$ carrier using (a) DSB, (b) SSB, (c) OCS, and (d) the proposed scheme. They are detected at back-to-back, after propagation of $20-\mathrm{km}$ SMF.

Then, we studied the second category of mm-wave generation, as shown in Fig. 12. The DSB signal was generated via an MZM which was driven by an electrical mixed signal of the baseband $10-\mathrm{Gb} / \mathrm{s}$ NRZ and the $80-\mathrm{GHz}$ clock, as shown in Fig. 12(a). The SSB signal was generated by using an offset optical bandpass filter (OBF) (Gaussian shaped, 20-GHz (a)

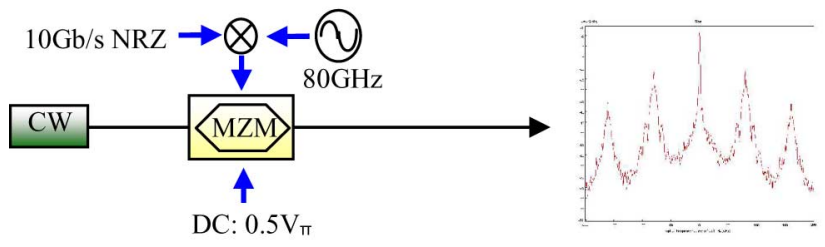

(b)

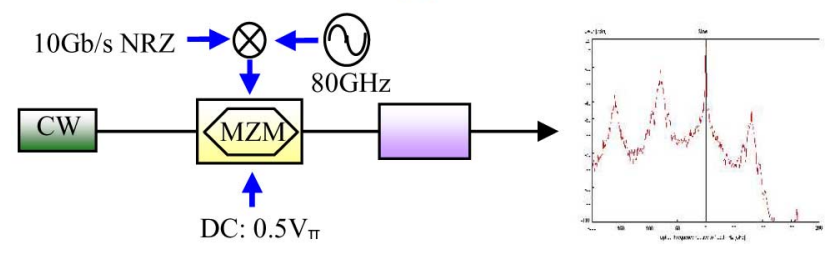

Fig. 12. Up-conversions of the DSB and SSB using RF mixer. (a) DSB. (b) SSB.

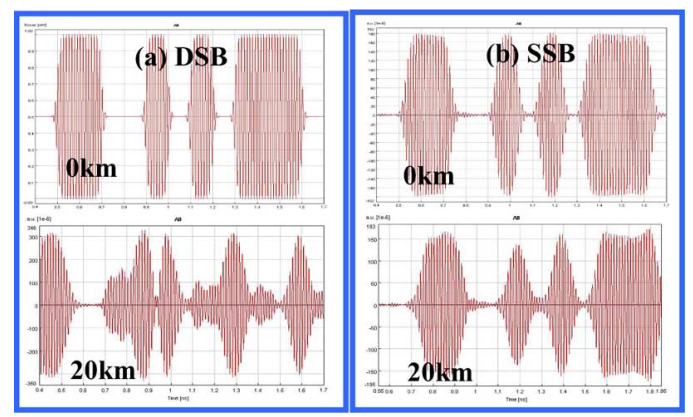

Fig. 13. Simulated time traces (second category) of 10-Gb/s NRZ mm-wave signal modulated by $80-\mathrm{GHz}$ carrier using (a) DSB and (b) SSB. They are detected at back-to-back, after propagation of $20-\mathrm{km}$ SMF.

3-dB bandwidth, offset by $120 \mathrm{GHz}$ ) to suppress one sideband $(>20 \mathrm{~dB})$ of the optical DSB signal, as shown in Fig. 12(b). Fig. 13 shows the simulated time traces of the optical mm-wave signal generated in the second category using RF mixer. We can observe that the DSB signal [Fig. 13(a)] also suffers from both fading and the code time shifting. However, the SSB signal generated by using a RF mixer and an offset OBF [Fig. 13(b)] performs much better than that in the first category (using cascaded MZMs) [Fig. 10(b)]. The SSB generated using an RF mixer and offset OBF performs as well as the proposed scheme. By studying the optical spectrum of the SSB signal generated in the second category, we can observe that the center wavelength is mainly dc component [Fig. 12(b)]. It is different from the case of SSB generated by using cascaded MZMs [Fig. 10(b)], which hence suffers form code time shifting.

\section{CONCLUSION}

Colorless RAU with optical carrier generated and distributed from the HE is an attractive network architecture. We proposed and demonstrated a novel bidirectional ROF system using a reference signal distribution to mitigate signal fading and code time shifting. Experimental characterization of the RSOA-based remodulation unit and the $40-\mathrm{GHz}$ optical reference signal is also performed. A signal remodulation scheme using downstream DPSK and upstream OOK was demonstrated in a $20-\mathrm{km}$ reach 
ROF system. Numerical simulations were performed to compare the proposed scheme with DSB, OCS, and SSB. Simulation results show that the SSB generated using an RF mixer and offset OBF performs as well as the proposed scheme. The proposed scheme requires dual-wavelength laser or other PLL, and this may increase the complexity and the cost of the system. We believe that the proposed scheme using separate data and reference signals could be an alternative to mitigate fiber chromatic dispersion, particularly when the mm-wave frequency is becoming higher and higher, and the cost of the high-frequency RF mixer will be much higher than the cost of the PLL.

\section{REFERENCES}

[1] T. Kamisaka, T. Kuri, and K. Kitayama, "Simultaneous modulation and fiber-optic transmission of $10 \mathrm{~Gb} / \mathrm{s}$ baseband and $60 \mathrm{GHz}$ band radio signals on a single wavelength," IEEE Trans. Microw. Theory Tech., vol. 49, no. 10, pp. 2013-2017, Oct. 2001.

[2] K. Ikeda, T. Kuri, and K. Kitayama, "Simultaneous three band modulation and fiber-optic transmission of $2.5 \mathrm{~Gb} / \mathrm{s}$ baseband, microwave, and $60 \mathrm{GHz}$ band signals on a single wavelength," J. Lightw. Technol., vol. 21, no. 12, pp. 3194-3202, Dec. 2003.

[3] A. Martinez, V. Polo, and J. Marti, "Simultaneous baseband and RF optical modulation scheme for feeding wireless and wireline heterogeneous access network," IEEE Trans. Microw. Theory Tech., vol. 49, no. 10, pp. 2018-2024, Oct. 2001.

[4] G. K. Chang, J. Yu, Z. Jia, and J. Yu, "Novel optical-wireless access network architecture for simultaneously providing broadband wireless and wired services," in Proc. Opt. Fiber Commun., Anaheim, CA, 2006, Paper OFM1.

[5] C. T. Lin, W. R. Peng, P. C. Peng, J. Chen, C. F. Peng, B. S. Chiou, and S. Chi, "Simultaneous generation of baseband and radio signals using only one single electrode Mach-Zehnder modulator with enhanced linearity," IEEE Photon. Technol. Letts., vol. 18, no. 23, pp. 2481-2483, Dec. 1, 2006.

[6] C. T. Lin, J. Chen, P. C. Peng, C. F. Peng, W. R. Peng, B. S. Chiou, and S. Chi, "Hybrid optical access network integrating fiber-to-the-home and radio-over-fiber systems," IEEE Photon. Technol. Letts., vol. 19, no. 8, pp. 610-612, Apr. 15, 2007.

[7] D. B. Payne and R. P. Davey, "The future of fibre access systems?," BT Technol. J., vol. 20, no. 4, pp. 104-114, 2002.

[8] G. Talli and P. D. Townsend, "Hybrid DWDM-TDM long reach PON for next generation optical access," J. Lightw. Technol., vol. 24, no. 7, pp. 2827-2834, Jul. 2006.

[9] S. B. Park, D. K. Jung, D. J. Shin, H. S. Shin, I. K. Yun, J. S. Lee, Y. K. Oh, and Y. J. Oh, "Colorless operation of WDM-PON employing uncooled spectrum-sliced reflective semiconductor optical amplifiers," IEEE Photon. Technol. Letts., vol. 19, no. 4, pp. 248-250, Feb. 15, 2007.

[10] F. Payoux, P. Chanclou, M. Moignard, and R. Brenot, "Gigabit optical access using WDM PON based on spectrum slicing and reflective SOA," in Proc. Eur. Conf. Opt. Commun., Glasgow, U.K., 2005, Paper We3.3.5.

[11] K. L. Lee and E. Wong, "Directly-modulated self-seeding reflective SOAs in WDM-PONs: Performance dependence on seeding power and modulation effect," in Proc. Eur. Conf. Opt. Commun., Cannes, France, 2006, Paper Tu4.5.2.

[12] L. Y. Chan, C. K. Chan, D. T. K. Tong, F. Tong, and L. K. Chen, "Upstream traffic transmitter using injection-locked Fabry-Perot laser diode as modulaotr for WDM access networks," Electron. Letts., vol. 38 , no. 1, pp. 43-45, 2002.

[13] W. Hung, C. K. Chan, L. K. Chen, and F. Tong, "An optical network unit for WDM access networks with downstream DPSK and upstream remodulated OOK data using injection-locked FP laser," IEEE Photon. Technol. Letts., vol. 15, no. 10, pp. 1476-1478, Oct. 2003.

[14] J. J. Vegas Olmos, T. Kuri, and K.-I. Kitayama, "Dynamic reconfigurable WDM $60 \mathrm{GHz}$ millimeter-wave-band radio-over-fiber access network: Architectural considerations and experiment," J. Lightw. Technol., vol. 25, no. 11, pp. 3374-3380, Nov. 2007.

[15] M. Huchard, M. Weiss, A. Pizzinat, S. Meyer, P. Guignard, and B. Charbonnier, "Ultra-broadband wireless home network based on 60-GHz WPAN cells interconnected via RoF," J. Lightw. Technol., vol. 26, no. 15, pp. 2364-2372, Aug. 1, 2008.
[16] J. Kim, Y.-D. Chung, K.-S. Choi, D.-S. Shin, J.-S. Sim, and H.-K. Yu, "60-GHz system-on-packaging transmitter for radio-over-fiber applications," J. Lightw. Technol., vol. 26, no. 15, pp. 2379-2387, Aug. 1, 2008.

[17] M. Weiß, M. Huchard, A. Stöhr, B. Charbonnier, S. Fedderwitz, and D. S. Jäger, "60-GHz photonic millimeter-wave link for short- to mediumrange wireless transmission up to $12.5 \mathrm{~Gb} / \mathrm{s}$," J. Lightw. Technol., vol. 26, no. 15, pp. 2424-2429, Aug. 1, 2008.

[18] J. J. V. Olmos, T. Kuri, and K.-I. Kitayama, "Dynamic reconfigurable WDM $60 \mathrm{GHz}$ millimeter-wave-band radio-over-fiber access network: architectural considerations and experiment," J. Lightw. Technol., vol. 25, no. 11 , pp. 3374-3380, Nov. 2007.

[19] J. J. V. Olmos, T. Kuri, T. Sono, K. Tamura, H. Toda, and K.-I. Kitayama, "Reconfigurable $2.5-\mathrm{Gb} / \mathrm{s}$ baseband and $60-\mathrm{GHz}(155-\mathrm{Mb} / \mathrm{s})$ millimeter-waveband radio-over-fiber (interleaving) access network," J. Lightw. Technol., vol. 26, no. 15, pp. 2506-2512, Aug. 1, 2008.

[20] A. Hirata, H. Takahashi, R. Yamaguchi, T. Kosugi, K. Murata, T. Nagatsuma, N. Kukutsu, and Y. Kado, "Transmission characteristics of 120-GHz-band wireless link using radio-on-fiber technologies," $J$. Lightw. Technol., vol. 26, no. 15, pp. 2338-2344, Aug. 1, 2008.

[21] M. Sauer, A. Kobyakov, and J. George, "Radio over fiber for picocellular network architectures," J. Lightw. Technol., vol. 25, no. 11, pp. 3301-3320, Nov. 2007.

[22] K.-I. Kitayama, A. Stöhr, T. Kuri, R. Heinzelmann, D. Jäger, and Y Takahashi, "An approach to single optical component antenna base stations for broadband millimeter-wave fiber-radio access systems," IEEE Trans. Microw. Theory. Tech., vol. 48, no. 12, pp. 2588-2595, Dec. 2000.

[23] J. Yu, Z. Jia, T. Wang, and G. K. Chang, "A novel radio-over-fiber configuration using optical phase modulator to generate an optical $\mathrm{mm}$-wave and centralized lightwave for uplink connection," IEEE Photon. Technol. Lett., vol. 19, no. 3, pp. 140-142, Feb. 2007.

[24] N. J. Gomes, A. Nkansah, and D. Wake, "Radio-over-MMF techniques-Part I: RF to microwave frequency systems," J. Lightw. Technol., vol. 26, no. 15, pp. 2388-2395, Aug. 1, 2008.

[25] A. M. J. Koonen and M. G. Larrodé, "Radio-over-MMF techniques-Part II: Microwave to millimeter-wave systems," J. Lightw. Technol., vol. 26, no. 15, pp. 2396-2408, Aug. 1, 2008.

[26] Z. Jia, J. Yu, G. Ellinas, and G. K. Chang, "Key enabling technologies for optical-wireless networks: Optical millimeter-wave generation, wavelength reuse, and architecture," J. Lightw. Technol., vol. 25 , no. 11, pp. 3452-3471, Nov. 2007

[27] M.-F. Huang, J. Yu, Z. Jia, and G.-K. Chang, "Simultaneous generation of centralized lightwaves and double/single sideband optical millimeter-wave requiring only low-frequency local oscillator signals for radio-over-fiber systems," J. Lightw. Technol., vol. 26, no. 15, pp. 2653-2662, Aug. 1, 2008.

[28] J. Yu, M. F. Huang, Z. Jia, T. Wang, and G. K. Chang, "A novel scheme to generate single-sideband millimeter-wave signals by using low-frequency local oscillator signal," IEEE Photon. Technol. Lett., vol. 20, no. 7, pp. 478-480, Apr. 2008

[29] J. Ma, J. Yu, C. Yu, X. Xin, J. Zeng, and L. Chen, "Fiber dispersion influence on transmission of the optical millimeter-wave generated using LN-MZM intensity modulation," J. Lightw. Technol., vol. 25, no. 11, pp. 3244-3256, Nov. 2007

[30] C.-T. Lin, Y.-M. Lin, J. Chen, S.-P. Dai, P. T. Shih, P.-C. Peng, and S Chi, "Optical direct-detection OFDM signal generation for radio-overfiber link using frequency doubling scheme with carrier suppression," Opt. Express, vol. 16, no. 9, pp. 6056-6063, 2008.

[31] K. Y. Cho, Y. Takushima, and Y. C. Chung, "10-Gb/s operation of RSOA for WDM PON," IEEE Photon. Technol. Lett., vol. 20, no. 18, pp. 1533-1535, Sep. 2008.

[32] L. Chen, Y. Shao, X. Lei, H. Wen, and S. Wen, "A novel radio-over-fiber system with wavelength reuse for upstream data connection," IEEE Photon. Technol. Letts., vol. 19, no. 6, pp. 387-389, Mar. 15, 2007.

[33] J. Yu, M. F. Huang, D. Qian, L. Chen, and G. K. Chang, "Centralized lightwave WDM-PON employing 16-QAM intensity modulated OFDM downstream and OOK modulated upstream signals," IEEE Photon. Technol. Lett., vol. 20, no. 18, pp. 1545-1547, Sep. 2008.

[34] C. W. Chow, "Wavelength remodulation using DPSK down-and-upstream with high extinction ratio for 10-Gb/s DWDM-passive optical networks," IEEE Photon. Technol. Lett., vol. 20, no. 1, pp. 12-14, Jan. 2008.

[35] C. H. Yeh, C. W. Chow, F. Y. Shih, C. H. Wang, Y. F. Wu, and S. Chi, "Tunable dual-wavelength fiber laser using optical-injection Fabry-Perot laser," IEEE Photon. Technol. Lett., vol. 20, no. 24, pp. 2093-2095, Dec. 2008. 


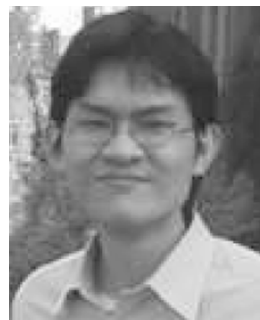

C. W. Chow (S'02-M'04) received the B.Eng. (firstclass honors) and Ph.D. degrees from the Department of Electronic Engineering, The Chinese University of Hong Kong, Shatin, Hong Kong, in 2001 and 2004, respectively. His Ph.D. focused on photonic packet switched networks.

He was a Postdoctoral Fellow at The Chinese University of Hong Kong, working on hybrid integration of photonic devices and silicon photonics. Between 2005 and 2007, he was a Postdoctoral Research Scientist at the Tyndall National Institute and Department of Physics, University College Cork (UCC), Ireland, working mainly on two European Union Projects: PIEMAN and TRIUMPH. In 2007, he joined the Department of Photonics, National Chiao Tung University, Hsinchu, Taiwan, as an Assistant Professor. His research interests are passive optical networks, radio-over-fiber, and photonic signal processing.

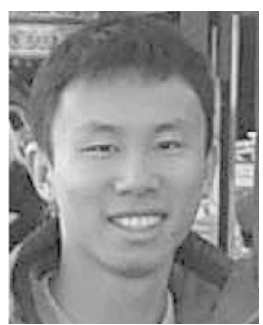

L. Xu (S'08) is currently working towards the Ph.D. degree at the Department of Electronic Engineering, The Chinese University of Hong Kong, Shatin, Hong Kong.

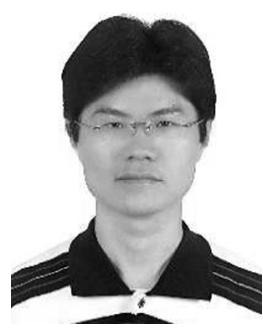

C. H. Yeh received the Ph.D. degree from the Institute of Electro-Optical Engineering, National Chiao Tung University, Hsinchu, Taiwan, in 2004.

In 2004, he joined the Information and Communications Research Laboratories (ICL), Industrial Technology Research Institute (ITRI), Hsinchu, Taiwan, as a Researcher, where in 2008, he was promoted to a Senior Researcher. His research interests are optical fiber communications, fiber lasers, fiber amplifiers, and wireless/fiber access network technologies.

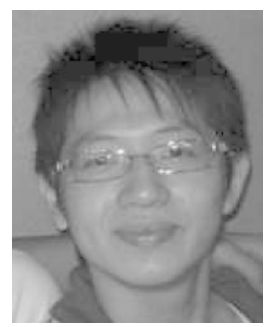

C. H. Wang (S'09) is currently working towards the $\mathrm{Ph} . \mathrm{D}$. degree at the Institute of Electro-Optical Engineering, National Chiao Tung University, Hsinchu, Taiwan.

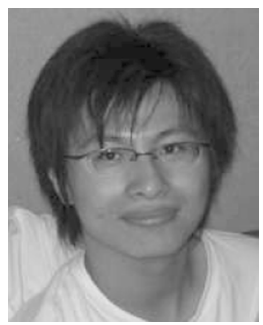

F. Y. Shih (S'09) is currently working towards the $\mathrm{Ph} . \mathrm{D}$. degree at the Institute of Electro-Optical Engineering, National Chiao Tung University, Hsinchu, Taiwan.

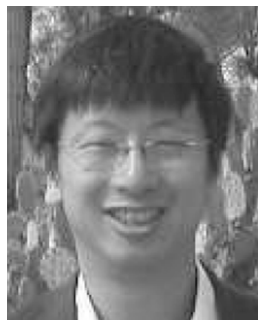

H. K. Tsang (M'90-SM'02) received the B.A. (honors) degree in engineering (electrical and information sciences), in 1987, and the Ph.D. and M.A. degrees in 1991, all from the University of Cambridge, Cambridge, U.K.

$\mathrm{He}$ worked on Quantum Confined Stark Effect waveguide modulators as a visitor at Bell Communications Research Inc. (Bellcore) in 1990. Between 1991 and 1993, he was a SERC Postdoctoral Fellow at the University of Bath. He joined The Chinese University of Hong Kong, Shatin, Hong Kong, in 1993, as a Lecturer in the Department of Electronic Engineering. In 2001, he was appointed Director of ASOC Technology at Bookham Technology plc, U.K. He returned to The Chinese University of Hong Kong in 2003. He has published over 200 papers in journals or conference proceedings.

Dr. Tsang is a former chair of the IEEE LEOS Hong Kong Chapter and currently serves as an Associate Editor of the IEEE LEOS NEWSLETTER as well as on the technical program committees of various IEEE, the Optical Society of America (OSA), and the International Society for Optical Engineering (SPIE) conferences.

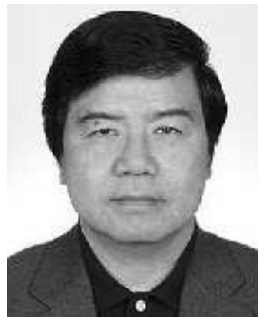

C. L. Pan (M'88-SM'03) received the Ph.D. degree in physics from Colorado State University, Ft. Collins, in 1979.

He is currently Tsing Hua Chair at the Department of Physics, National Tsing Hua University (NTHU), Hsinchu, Taiwan. Prior to joining NTHU in February 2009, he was University Chair Professor at the Department of Photonics (DOP) and Institute of Electro-Optical Engineering (IEO), National Chiao Tung University (NCTU), Hsinchu, Taiwan. He was the Director of IEO, NCTU (1992-1995), founding Chair of the DOP (2004-2006). He has also taken sabbatical leaves at the University of California, Berkeley, Osaka University, Japan, and The Chinese University of Hong Kong, Shatin, Hong Kong. His research interests are lasers and their applications in broadband optical communication, precision metrology, ultrafast, and $\mathrm{THz}$ Photonics. In particular, his group has recently developed numerous devices for laser and $\mathrm{THz}$ photonics with liquid crystal enabled functionalities.

Prof. Pan is a member of the Phi Tau Phi Honor Society (1991) and a Fellow of the Photonic Society of Chinese Americans (1998), the Optical Society of America (OSA) (2004), The International Society of Optical Engineering (SPIE) (2004), and the Physical Society of Republic of China (2005). He was presented Merit Research Fellow Award of the National Science Council (2002), the Academics Award of the Ministry of Education (2004), the Engineering Medal by the Optical Engineering Society, Taiwan (2004), Outstanding Engineering Professor Award of the Chinese Institute of Engineers (2006), and the Pan Wen Yuan Foundation Research Excellence Award (2007)

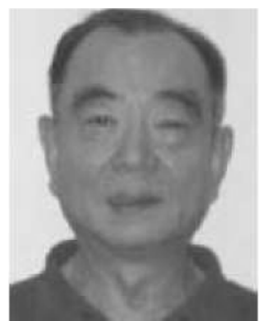

S. Chi received the Ph.D. degree in electrophysics from the Polytechnic Institute of Brooklyn, New York, in 1971.

He joined the faculty of National Chiao Tung University, Hsinchu, Taiwan, where he is currently a Professor of Electro-Optical Engineering. From 1993 to 1996, he received the Distinguished Research Award sponsored by the National Science Council, Taiwan. Since 1996, he has been the Chair Professor of the Foundation for Advancement of Outstanding Scholarship. His research interests are optical fiber communications, optical solitons, and optical fiber amplifiers.

Dr. Chi is a Fellow of the Optical Society of America (OSA) and the Photonics Society of Chinese-Americans. 